Vol. 6(5), 21-28 (2013)

doi: $10.4067 / S 0718-50062013000500004$

\title{
Jerarquización de Competencias específicas en el programa de Física de la Escuela Superior de Física y Matemáticas del IPN-México utilizando la matriz de Morganov-Heredia
}

\author{
Mario H. Ramírez ${ }^{(1)}$, Arturo F. Méndez-Sánchez ${ }^{(2)}$, Leonor Pérez-Trejo ${ }^{(2)}$, Miguel Olvera-Aldana ${ }^{(3)}$ \\ (1) Departamento de Posgrado en Física Educativa, Centro de Investigación en Ciencia Aplicada y \\ Tecnología Avanzada, Instituto Politécnico Nacional, Av. Legaria 694, Col. Irrigación, Del. Miguel Hidalgo, \\ CP 11500, México D.F.- México (mramirezd@ipn.mx) \\ (2) Departamento de Física, Escuela Superior de Física y Matemáticas, Instituto Politécnico Nacional, \\ Edificio 9, UPALM, Zacatenco, CP 07738, México, D.F.- México (arturo@esfm.ipn.mx; leopt@esfm.ipn.mx) \\ (3) Departamento de Formación Básica, Escuela Superior de Cómputo, Instituto Politécnico Nacional, Ave. \\ Juan de Dios Batíz s/n esquina Miguel Othón de Mendizabal, UPALM, Lindavista, CP 07738, México, D.F.- \\ México (molveraa@ipn.mx)
}

Recibido May. 06, 2013; Aceptado Jun. 17, 2013; Versión final recibida Ago. 09, 2013

\begin{abstract}
Resumen
En este trabajo, se utilizó la llamada matriz de Morganov-Heredia para obtener una jerarquización de las competencias específicas para el graduado en Física de la Licenciatura en Física y Matemáticas en la Escuela Superior de Física y Matemáticas del Instituto Politécnico Nacional en México. El estudio se enmarca en los esfuerzo de la institución para implantar el Modelo Educativo por Competencias en sus planes y programas de estudio. El método Morganov-Heredia permitió identificar y agrupar competencias por grupo de características, tales como competencias laborales, disciplinares y sociales. Además la matriz permitió ordenar las competencias propuestas desde la más básica hasta la competencia meta final.
\end{abstract}

Palabras clave: aprendizaje de la física, modelo educativo por competencias, jerarquización de competencias, matriz de Morganov-Heredia

\section{Hierarchy of Competencies in the Physics program at the Higher School of Physics and Mathematics of the IPN in Mexico using the Morganov-Heredia matrix}

\begin{abstract}
In this paper, the so-called Morganov-Heredia matrix was employed to obtain a hierarchy of competencies for physics graduates in Physics at the Higher Scholl of Physics and Mathematics of the National Polytechnic Institute in Mexico. The study is done in the context of the effort of the institution to implement Competence-Based Educational Model on its curricula. The Morganov-Heredia matrix allowed identifying and grouping competences by their characteristics, such as labor disciplinary and social competences. Also, the matrix allowed organizing the proposed competences from the most basic one to the final competence goal.
\end{abstract}

Keywords: physics learning, competency-based educational model, competence hierarchy, MorganovHeredia matrix 


\section{INTRODUCCIÓN}

La enseñanza basada en competencias establece la posibilidad de organizar los contenidos de aprendizaje en unidades de significado completo, resolviendo un problema a la vez, con fuertes interrelaciones entre sus componentes. Esta característica permite el diseño de módulos secuenciados e integrados para el desarrollo de las competencias (Sánchez, 2012).

En los últimos años se ha incorporado la enseñanza por competencias a las instituciones de educación superior, tanto en México como a nivel internacional (Argudín, 2005; De Asís 2007). En este sentido se han hecho esfuerzos por estudiar las competencias específicas a desarrollar en diferentes programas universitarios por diversas instancias, uno de estos estudios ha sido llevado a cabo por el Proyecto Tuning, tanto en Europa como en América Latina. De manera particular, el proyecto Tuning América Latina estudió en su capítulo 4.6 los programas de grado en carreras de Física en 12 países que incluían a México. En este informe se reportan 22 competencias específicas para las carreras de Física, la cuales se obtuvieron por consenso de profesores de los 12 países participantes. El informe Tuning también reporta cuales competencias fueron valoradas como importantes, cuál fue el rango de realización de dichas competencias y cuáles son las competencias claves a desarrollar, todo lo anterior obtenido por la opinión de cuatro agentes: Académicos, Estudiantes, Egresados y Empleadores (Beneitone, 2007).

En México, la realidad de la implementación de este modelo ha tenido una serie de problemas en las escuelas, debido a que no existe una manera general de llevar a cabo las recomendaciones hechas por organismos internacionales como la OCDE en relación a los programas educativos por competencias (Hernández y Rodríguez, 2008). Ejemplo de lo anterior son el IPN en la mayoría de sus programas (IPN, 2004), la Universidad de Zacatecas, Universidad Autónoma de Coahuila, Instituto Tecnológico y de Estudios Superiores de Monterrey, Benemérita Universidad Autónoma de Puebla y la Universidad Autónoma de Baja California lo han implementado específicamente en su programa de Física. Proyectos como Tuning realizan recomendaciones generales acerca de las competencias específicas a desarrollar en los graduados de diferentes disciplinas, no obstante (al menos en su capítulo de Física) dejan preguntas abiertas tales como: ¿son competencias de tipo puntual o transversal?, ¿con qué área de la Física están relacionadas y en qué nivel?, ¿cómo puede evaluarse el aprendizaje de estas competencias?, ¿cómo puede establecerse el tiempo de aprendizaje de estas competencias? y ¿de qué manera deben modificarse los métodos de enseñanza para favorecer el aprendizaje de estas competencias? En el caso de México el estudio lo coordinó la Universidad de Sonora.

A pesar de lo anterior, existen esfuerzos para implementar las competencias propuestas por el Proyecto Tuning en el área de la Física a nivel bachillerato (Aguilera y Cuevas, 2011; Ramírez y Olvera, 2012). A nivel universitario, en México se ha incorporado a las facultades de ciencias y escuelas de Física poco a poco la idea de desarrollar competencias específicas, sin embargo la percepción acerca de estas ha sido un obstáculo para su mayor éxito. En la ESFM-IPN se ha investigado la percepción que los profesores del programa de Física tienen acerca del modelo por competencias como un primer paso para su posible desarrollo (Pérez-Trejo et al., 2012; González y Rasilla, 2011). Sin embargo, el solo obtener las competencias específicas para un graduado en carreras de Física resulta insuficiente para el profesor encargado de desarrollarlas (Cañas, 2007). Es necesario establecer un orden o prioridad para que las competencias puedan ser construidas por los profesores. En el caso de México la Secretaría de Educación Pública (SEP) es la encargada de supervisar y aprobar todos los planes y programas de estudios en el país, por lo que sus disposiciones y ordenamientos se tienen que acatar en todos los niveles educativos. Para la jerarquización de las competencias, la SEP establece desde el año 2010 el uso de la llamada Matriz de Morganov-Heredia. De lo anterior en este trabajo partimos de la pregunta de investigación: Tomando como base las competencias propuestas por el Proyecto Tuning, ¿cuál es el resultado de utilizar la Matriz de Morganov-Heredia para la jerarquización de competencias específicas por los profesores de la ESFM-IPN?

Nuestra hipótesis de trabajo inicial para dar respuesta a esta pregunta fue que utilizando como herramienta la Matriz de Morganov-Heredia se logra una eficiente jerarquización de las competencias específicas por parte de los profesores de la ESFM-IPN y permite identificar y agrupar competencias por grupo de características como competencias laborales, disciplinares o sociales. Además, está matriz permitirá a partir de una serie de pasos, ordenar las competencias propuestas desde la más básica hasta la competencia meta final.

\section{HISTORIA, MISIÓN Y VISIÓN DE LA ESFM-IPN}

Es importante contextualizar el problema desde tres tópicos que se consideran importantes para esta investigación: la historia, misión y visión de la ESFM-IPN (ESFM, 2013).Desde su creación la ESFM-IPN tuvo como objetivos fijos la de formar especialistas para la docencia en Física y Matemáticas, para la 
investigación científica y tecnológica, y para contribuir al desarrollo de la industria nacional. La ESFM-IPN es la escuela del Instituto Politécnico Nacional creada para formar integralmente profesionales de excelencia en el campo de las ciencias físico-matemáticas, con alto grado de compromiso y responsabilidad social, capaces de contribuir al desarrollo económico, científico y tecnológico del país; mediante la docencia, la investigación, la innovación, la vinculación y la divulgación del conocimiento en estas áreas (Camarena, 2006).

La ESFM es líder en su ámbito, cuenta con un modelo educativo flexible, centrado en el aprendizaje, que desarrolla la capacidad creativa, la actitud innovadora, el trabajo cooperativo y los hábitos de tenacidad en sus alumnos para que sean competitivos a nivel internacional. Sus profesores poseen una alta formación académica, comprometidos con la labor docente; la investigación que realizan contribuye al desarrollo de las ciencias físico-matemáticas, aplicándolas para la solución de problemas de alto impacto social, además, difunden y promueven los avances tecnológicos y científicos.

El Instituto Politécnico Nacional, está viviendo un periodo de transición en todas sus estructuras y la académica no es la excepción, la cual ha tratado de impulsar el modelo de enseñanza a través de competencias. Para este fin se han impartido una serie de diplomados y talleres desde el año 2000. Bajo este contexto se realizaron una serie de cuestionamientos a catedráticos de la ESFM-IPN, sobre el modelo basado en competencias. Es claro que la realidad actual de la docencia implica un esfuerzo mayor en la actualización y formación de los profesores, más allá del conocimiento de su propia disciplina. Esta situación es más complicada para el área de las llamadas "ciencias duras" (entendiendo como tales para este trabajo a la Física, las Matemáticas, la Biología y la Química) dada la resistencia de los profesores a la incorporación de elementos más allá de la enseñanza tradicional.

\section{METODOLOGÍA}

En esta sección se muestra lo referente a las competencias más importantes atendidas en la ESFM-IPN como el núcleo básico de trabajo. En el caso de Física, los autores de este trabajo tomamos como base las competencias sugeridas a desarrollar por el Proyecto Tuning América Latina (Beneitone, 2007, Deusto, 2008) para el graduado en carreras de Física (Físicos, Ingenieros Físicos, Biofísicos, Físicos Aplicados, etc.). Lo anterior se debe a que en México, y en el IPN como caso de estudio particular, no existe la Licenciatura de Profesor de Física o afín para nivel bachillerato o universitario (como si es el caso de Colombia, Brasil, Argentina y otros países latinoamericanos) por lo que un buen porcentaje de los profesores dedicados a enseñar Física en estos niveles son de origen, profesionales de la misma. En segundo lugar, El Proyecto Tuning ya considera dentro de las 22 competencias específicas propuestas una división de las mismas que llama "competencias cognitivas" lo que permitió a su vez complementarlas por las que se consideraron importantes a desarrollar para un profesional que se dedicará al ejercicio de la Física, a saber:

1. Plantear, analizar y resolver problemas físicos, tanto teóricos como experimentales, mediante la utilización de métodos analíticos, experimentales o numéricos.

2. Construir modelos simplificados que describan una situación compleja, identificando sus elementos esenciales y efectuando las aproximaciones necesarias.

3. Utilizar o elaborar programas o sistemas de computación para el procesamiento de información, cálculo numérico, simulación de procesos físicos o control de experimentos.

4. Verificar y evaluar el ajuste de modelos a la realidad, identificando su dominio de validez.

5. Aplicar el conocimiento teórico de la Física en la realización e interpretación de experimentos.

6. Demostrar una comprensión profunda de los conceptos y principios fundamentales, tanto de la Física clásica como de la Física moderna.

7. Describir y explicar fenómenos naturales y procesos tecnológicos en términos de conceptos, principios y teorías Físicas.

8. Desarrollar argumentaciones válidas en el ámbito de la Física, identificando hipótesis y conclusiones.

9. Sintetizar soluciones particulares, extendiéndolas hacia principios, leyes o teorías más generales.

10. Percibir las analogías entre situaciones aparentemente diversas, utilizando soluciones conocidas en la resolución de problemas nuevos.

11. Estimar el orden de magnitud de cantidades mensurables para interpretar fenómenos diversos.

12. Demostrar destrezas experimentales y uso de métodos adecuados de trabajo en el laboratorio.

13. Participar en actividades profesionales relacionadas con tecnologías de alto nivel, sea en el laboratorio o en la industria.

14. Participar en asesorías y elaboración de propuestas en ciencia y tecnología en temas con impacto económico y social en el ámbito nacional. 
15. Actuar con responsabilidad y ética profesional, manifestando conciencia social de solidaridad, justicia y respeto por el ambiente.

16. Demostrar hábitos de trabajo necesarios para el desarrollo de la profesión tales como el trabajo en equipo, el rigor científico, el autoaprendizaje y la persistencia.

17. Buscar, interpretar y utilizar información científica.

18. Comunicar conceptos y resultados científicos en lenguaje oral y escrito ante sus pares, y en situaciones de enseñanza y de divulgación.

19. Participar en la elaboración y desarrollo de proyectos de investigación en Física o interdisciplinarios.

20. Demostrar disposición para enfrentar nuevos problemas en otros campos, utilizando sus habilidades y conocimientos específicos.

21. Conocer y comprender el desarrollo conceptual de la Física en términos históricos y epistemológicos.

22. Conocer los aspectos relevantes del proceso de enseñanza-aprendizaje de la Física, demostrando disposición para colaborar en la formación de científicos.

A partir de estas 22 competencias se preguntó a los profesores de la licenciatura en Física y Matemáticas de la ESFM-IPN ¿Cuáles de estas competencias consideraban las más importantes a desarrollar en los estudiantes?, esta pregunta se pudo contestar vía electrónica en el sitio: http://www.physics-educationipn.com.mxl; los resultados se muestran en la siguiente gráfica (Fig. 1). Se encuestó a un grupo de 46 profesores, conformado por profesionales de la Física, de los cuales $90 \%$ son egresados de la licenciatura de Física y Matemáticas de la misma escuela y el resto de otras Instituciones Universitarias. La mayoría de los profesores está contratada de tiempo completo y se dedica exclusivamente a la docencia y a la investigación en la ESFM-IPN. Con las respuestas obtenidas se hizo un consenso entre el grupo de investigación, considerando como las más recurrentes aquellas con más de $80 \%$ de concordancia entre los profesores. De acuerdo a este ejercicio se obtuvieron 8 competencias específicas (Pérez-Trejo et al., 2012).

De la Fig. 1 se desprende que las ocho competencias elegidas como las más importantes son las competencias: $1,2,4,6,7,15,16$ y 20 . Por otro lado, no basta con conocer cuáles son las competencias consideradas más importantes sino que además es importante poder jerarquizarlas, es decir, ordenarlas de manera que se pueda tener una referencia sobre qué competencias serían base y cuales serían una "meta" final. Más allá de criterios subjetivos, como preferencias personales de los profesores, es importante mencionar que existe una herramienta que permite realizar esta jerarquización. Esta herramienta es conocida como matriz de Morganov-Heredia $(\mathrm{M}-\mathrm{H})$ y se detalla en la siguiente sección.

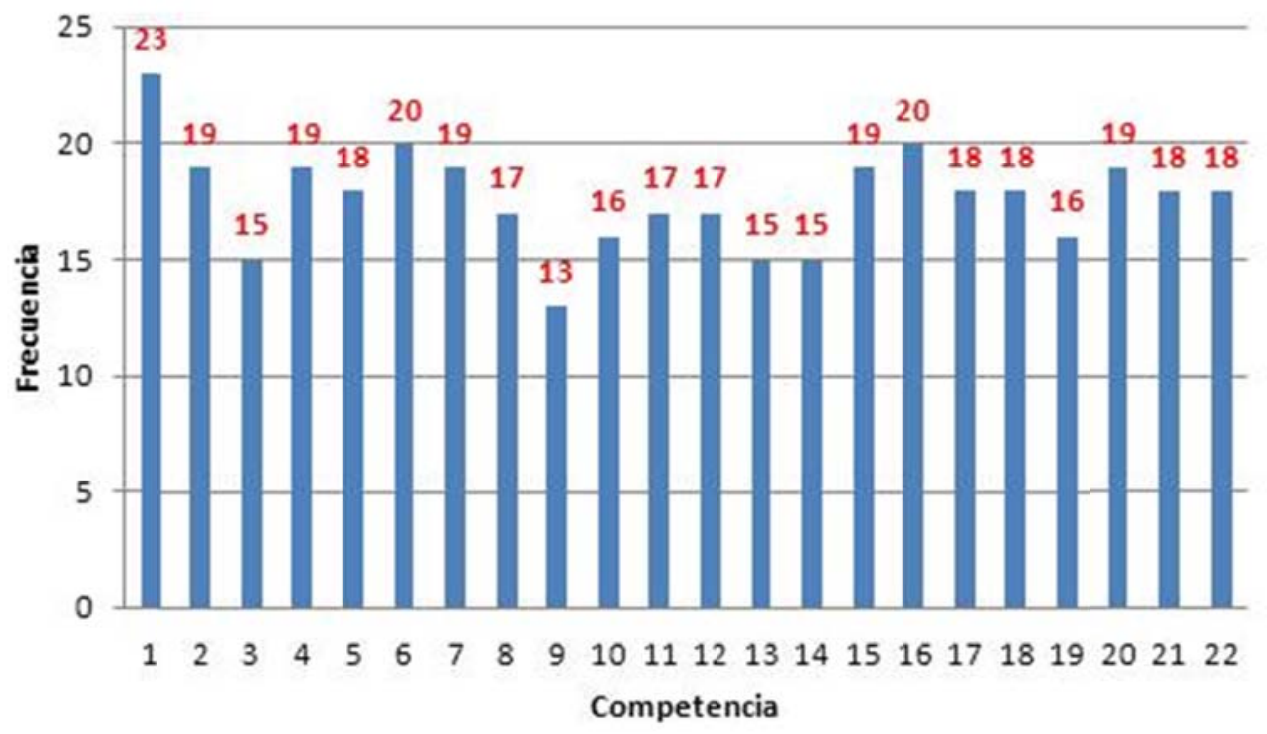

Fig. 1: Gráfico de Frecuencias, el número sobre la barra es la cantidad de profesores que consideraron importante la competencia respectiva.

\section{MATRIZ DE MORGANOV-HEREDIA}

En el Manual Metodológico de la Secretaría de Educación Pública de México (SEP, 2010) se propone que para dar un orden didáctico a las competencias específicas y poder a su vez vincularlas con los temas y subtemas de aprendizaje en una unidad de aprendizaje se haga por pares la siguiente pregunta: Para desarrollar la competencia $X$, ¿es necesario antes desarrollar la competencia $Y$ ? Con base en la pregunta anterior se ubican las competencias en un arreglo de renglones y columnas formando la matriz de $\mathrm{M}-\mathrm{H}$. En 
cada casilla de la matriz se considera la respuesta a la pregunta formulada tomando como "uno" una respuesta positiva y como "cero" el caso contrario. En el caso de ser la misma competencia, tanto en la fila como en la columna, no se hace comparación dado que ésta no puede ser requisito de sí misma. Finalmente se realiza la suma de unos de manera horizontal.

Para realizar la matriz M-H se conformó un grupo de investigación constituido por los autores de este trabajo con la formación profesional en Física, posgrados en Física Teórica y Educativa, con experiencia activa en investigación educativa y docencia. De manera que, utilizando las competencias ya numeradas como se señaló anteriormente y aplicando consenso entre el grupo de investigación se obtuvo la siguiente matriz (Fig. 2):

\begin{tabular}{|c|c|c|c|c|c|c|c|c|c|}
\hline $\mathbf{X}$ & $\mathbf{1}$ & $\mathbf{2}$ & $\mathbf{4}$ & $\mathbf{6}$ & $\mathbf{7}$ & $\mathbf{1 5}$ & $\mathbf{1 6}$ & $\mathbf{2 0}$ & $\begin{array}{c}\text { Suma } \\
\text { de } \\
\text { unos }\end{array}$ \\
\hline $\mathbf{1}$ & - & 1 & 1 & 0 & 1 & 0 & 0 & 1 & 4 \\
\hline $\mathbf{2}$ & 1 & - & 1 & 1 & 1 & 0 & 0 & 0 & 4 \\
\hline $\mathbf{4}$ & 1 & 0 & - & 1 & 1 & 0 & 0 & 0 & 3 \\
\hline $\mathbf{6}$ & 1 & 1 & 1 & - & 1 & 0 & 0 & 1 & 5 \\
\hline $\mathbf{7}$ & 1 & 1 & 0 & 1 & - & 0 & 0 & 1 & 4 \\
\hline $\mathbf{1 5}$ & 0 & 0 & 1 & 0 & 0 & - & 1 & 1 & 3 \\
\hline $\mathbf{1 6}$ & 0 & 0 & 0 & 0 & 1 & 0 & - & 1 & 2 \\
\hline $\mathbf{2 0}$ & 0 & 0 & 0 & 0 & 1 & 0 & 1 & - & 2 \\
\hline
\end{tabular}

Fig. 2: Matriz de Morganov-Heredia, el primer renglón y la primera columna corresponden a las competencias propuestas como más importantes.

Una vez obtenida la matriz de $\mathrm{M}-\mathrm{H}$, el siguiente paso para la jerarquización es contabilizar los "unos" en cada reglón para las competencias y ordenar entonces desde la competencia que tuvo el menor número de ellos al mayor para obtener el orden didáctico de desarrollo de las competencias. En el caso de que existan competencias con el mismo número de "unos", el Manual Metodológico propone que los especialistas decidan cual va primero. En nuestro caso el orden a partir de la matriz se muestra a continuación (Fig. 3).

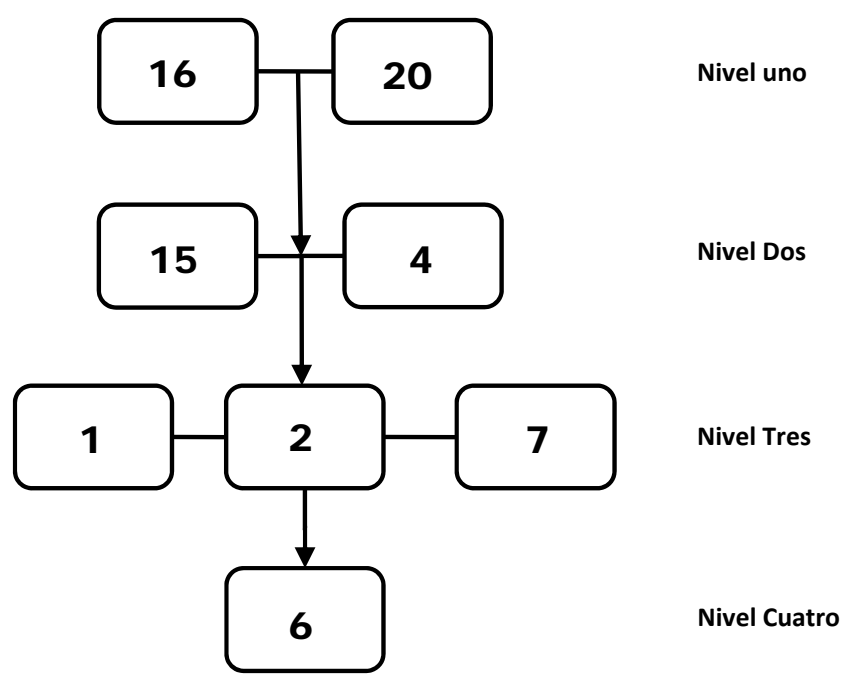

Fig.3: Diagrama obtenido a partir de la Matriz de Morganov-Heredia para jerarquizar las competencias propuestas.

Como se puede ver en la figura 2, las competencias 16 y 20; 15 y $4 ; 1,2$ y 7 tienen el mismo número de unos respectivamente, por lo que el grupo de investigación procedió como lo marca el Manual Metodológico, obteniendo finalmente la siguiente secuencia jerárquica (Fig. 4): 


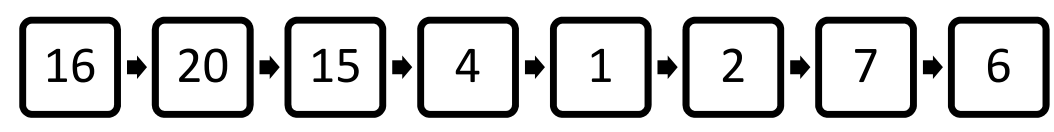

Fig. 4: Secuencia jerárquica final de las competencias.

La elección del orden de las competencias realizado por el grupo de investigación toma en cuenta nuestra participación y experiencia tanto como estudiantes egresados de programas de Física como de docencia en los mismos programas. En el nivel Uno del diagrama de la Fig. 3, se eligió para comenzar la secuencia la competencia 16 ya que (a criterio del grupo de investigación) es necesario inculcar los hábitos de trabajo necesarios para el desarrollo de la profesión, seguida de la competencia 20 que permite demostrar disposición para enfrentar problemas en otros campos. En el nivel Dos se escogió la competencia 15 que corresponde a actuar con responsabilidad y ética profesional seguida de la competencia 4 que consiste en verificar y evaluar el ajuste de modelos a la realidad. En el nivel Tres se escogió la competencia 1 donde se plantean, analizan y resuelven problemas físicos, seguida de la competencia 2, donde se construyen modelos simplificados que describan una situación compleja, posteriormente la competencia 7 que permite explicar fenómenos naturales y procesos tecnológicos a partir de los principios físicos. Finalmente en el nivel Cuatro la competencia 6 que consiste en demostrar una comprensión profunda de los principios fundamentales de la Física.

Al comparar éstas competencias y el ordenamiento sugerido con la sistematización de las competencias específicas realizadas por el proyecto Tuning América Latina, podemos ver que las primeras, 15,16 y 20 caen en el grupo III de las competencias laborales y sociales. Posteriormente, la 1 y la 2 caen en el grupo II de las competencias metodológicas. En este caso en particular, la 1 cae en la subcategoría de las competencias sistémicas, mientras que la 2 está en la subcategoría de las competencias instrumentales. Finalmente, la 6 y la 7 caen en el grupo de las competencias cognitivas. De este análisis se puede ver que el orden propuesto de las competencias evidencia también una secuencia a partir de la sistematización, la cual se puede ver en la figura 5, es decir, de manera general se propone primero desarrollar las competencias laborales y sociales seguidas por las competencias metodológicas y finalmente las competencias cognitivas.
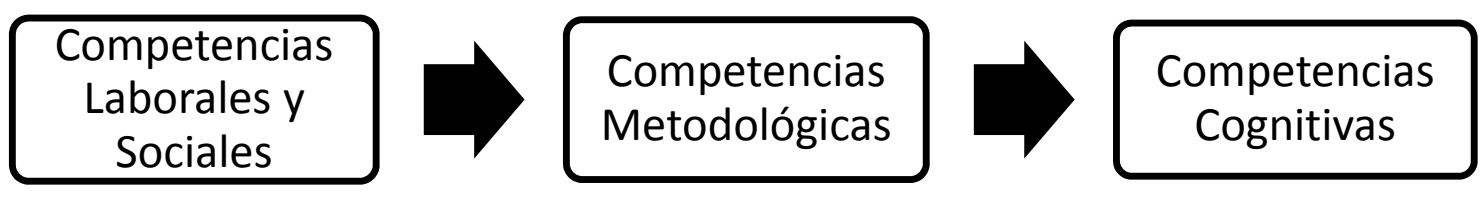

Fig. 5: Secuencia de desarrollo de competencias específicas en relación a sus categorías.

\section{DISCUSIÓN}

Es importante señalar que la matriz de M-H es un instrumento avalado por la SEP, la cual rige la educación en México, y permitió realizar la jerarquización de las competencias así como obtener una clasificación de categorías. La secuencia jerárquica de las competencias permite ver que para un graduado en Física es importante desarrollar habilidades, valores y actitudes en un orden que el Informe Tuning o los planes y programas de estudio de las Carreras de Física no especifican o proponen. En el caso de la presente propuesta se puede partir de una competencia "Base", que pertenece a la categoría de las competencias laborales y sociales, desarrollar competencias metodológicas hasta llegar a una competencia "Meta" de la categoría de las competencias cognitivas, es decir, en un plan de desarrollo de competencias específicas se podría procurar tener el siguiente orden:

- Demostrar hábitos de trabajo necesarios para el desarrollo de la profesión tales como el trabajo en equipo, el rigor científico, el autoaprendizaje y la persistencia.

- Demostrar disposición para enfrentar nuevos problemas en otros campos, utilizando sus habilidades y conocimientos específicos.

- Actuar con responsabilidad y ética profesional, manifestando conciencia social de solidaridad, justicia y respeto por el ambiente.

- Verificar y evaluar el ajuste de modelos a la realidad, identificando su dominio de validez.

- Plantear, analizar y resolver problemas físicos, tanto teóricos como experimentales, mediante la utilización de métodos analíticos, experimentales o numéricos. 
- Construir modelos simplificados que describan una situación compleja, identificando sus elementos esenciales y efectuando las aproximaciones necesarias.

- Describir y explicar fenómenos naturales y procesos tecnológicos en términos de conceptos, principios y teorías Físicas.

- Demostrar una comprensión profunda de los conceptos y principios fundamentales, tanto de la Física clásica como de la Física moderna.

Estas competencias se pueden desarrollar a lo largo de toda la carrera, de manera que la meta final del graduado en Física sea "Demostrar una comprensión profunda de los conceptos y principios fundamentales, tanto de la Física clásica como de la Física moderna", situación con la que estarían de acuerdo la gran mayoría de los profesores en las escuelas de Física.

Un punto importante a señalar es que esta jerarquización de competencias no es una propuesta de estrategia didáctica para la construcción y desarrollo de tales competencias en el aula, sino que muestra una metodología para la construcción de un orden en el desarrollo de las mismas. Por lo que no se detalla la manera de abordar las competencias desde la perspectiva de la jerarquización propuesta, ya que escapa a los objetivos de este trabajo. No obstante, actualmente la ESFM-IPN se encuentra en el rediseño de planes y programas de la licenciatura de Física y Matemáticas, donde además se está utilizando la metodología que aquí se propuso, lo cual implicará definir momentos, espacios y estrategias.

Tampoco se debe perder de vista que en este trabajo se realizó el ejercicio solo con 8 competencias elegidas como importantes y que para una jerarquización completa se deberían de tomar en cuenta las 22 competencias propuestas por el Proyecto Tuning. Otro aspecto, es que la consideración de qué competencias son las importantes para los profesores es subjetiva ya que se puede entrar en polémica acerca de ¿Por qué es importante tal o cual competencia?

\section{CONCLUSIONES}

El método Morganov-Heredia permitió identificar y agrupar competencias por grupo de características, tales como competencias laborales, disciplinares y sociales.

La matriz Morganov-Heredia permitió ordenar las competencias propuestas desde la más básica hasta la competencia meta final.

Los resultados presentados en este trabajo son parte de una investigación más amplia que incluye a instituciones de nivel superior con programas de Física en México cuyos resultados abarcan desde la percepción sobre el modelo por competencias, las competencias consideradas como más importantes y como más atendidas entre otros y que serán reportados posteriormente.

\section{REFERENCIAS}

Aguilera, J. y Cuevas, G. "Adaptación de la Metodología Tuning para el levantamiento del perfil de egreso de los alumnos de Bachillerato de la Universidad de los Andes", Calidad en la Educación, Número 34, 219230 (2011).

Argudín, Y. “Educación basada en competencias: nociones y antecedentes”. México, Editorial Trillas (2005).

Beneitone, P. "Reflexiones y perspectivas de la educación superior en América Latina, Informe Final Proyecto Tuning para América Latina 2004-2007", Bilbao, Publicaciones de la Universidad de Deusto (2007).

Benemérita Universidad Autónoma de Puebla www.fcfm.buap.mx Acceso: 6 de agosto (2013).

Camarena, G. P., Un enfoque de las Ciencias en Contexto desde la didáctica. Revista Innovación Educativa, 6(31), 21-31 (2006).

Cañas, A., Martín-Díaz, M. y Nieto, J. “Competencia en el conocimiento y la interacción con el mundo físico". Madrid, Alianza Editorial (2007).

De Asis Blas, F. “Competencias profesionales en la formación profesional”. Madrid. Alianza Editorial (2007). ESFM http://www.esfm.ipn.mx/Conocenos/Paginas/Mision.aspx Acceso: 18 de febrero (2013). 
Hernández, A. y Rodríguez, K. "La organización para la cooperación y el desarrollo económico, OCDE, Y la definición de competencias en educación superior: el caso de México". EDUCERE, Año 12, Núm. 43, 751758 (2008).

González, L.M. y Rasilla, M. "Una Estrategia para el Aprendizaje de la Cultura Científica". Formación Universitaria, Vol. 4, No 2, 15-26 (2011).

Instituto Tecnológico de Estudios Superiores de Monterrey http://www.itesm.edu/wps/wcm/connect/itesm/tecnologico+de+monterrey/carreras+profesionales/areas+de+ estudio/ingenieria+y+ciencias/ingeniero+fisico+industrial/monterrey+ifi Acceso: 6 de agosto (2013).

IPN. "Materiales para la reforma Libro 1: Un Nuevo modelo educativo para el IPN". Instituto Politécnico Nacional, México (2004).

Pérez-Trejo, L., Méndez-Sánchez, A., Ramírez, M., Olvera, M. y González, A. "Percepción de profesores de Física y Matemáticas sobre el modelo educativo basado en competencias". Lat. Am. J. Phys. Educ. Vol. 6, No. 4, 614-617 (2012).

Ramírez, M. y Olvera, M. "Formación de profesores de bachillerato en competencias específicas de la Física utilizando cursos intersemestrales en el IPN", Lat. Am. J. Phys. Educ. Vol. 6, No. 2, 284-291 (2012).

Sánchez, I. "Evaluación de una renovación metodológica para un aprendizaje significativo de la Física". Formación Universitaria, Vol. 5, No 5, 51- 65 (2012).

SEP. 105 DGCFT, SEP (2002) La Técnica de Morganov-Heredia para la organización secuencial de materias y contenidos en: Manual Metodológico y de Procedimientos para la Elaboración de Paquetería Didáctica. Educación Basada en Competencias. http://intranet.dgcft.sep.gob.mx/uploads/Manual\%20Metodol\%C3\%B3gico\%20y\%20de\%20Procedimientos\% 20para\%20la\%20Elaboraci\%C3\%B3n\%20de\%20Paqueter\%C3\%Ada\%20Did\%C3\%A1ctica.pdf. Acceso: 20 de agosto (2009).

Universidad Autónoma de Baja California

http://www.uabc.mx/formacionbasica/guiac/ensenada/historia_ciencias_ens.htm Acceso: 6 de agosto (2013).

Universidad Autónoma de Zacatecas http://fisica.uaz.edu.mx/portal/es/licenciatura/perfil Acceso: 6 de agosto (2013).

Universidad de Deusto. "Reference Points for the Design and Delivery of Degree Programmes in PHYSICS". Bilbao, Publicaciones de la Universidad de Deusto (2008). 